\title{
Comunicación y migración juvenil: un imaginario de futuro en Tijuana
}

\author{
Gerardo León Barrios \\ Universidad Autónoma de Baja California, \\ Campus Tijuana
}

La vida social contemporánea requiere, cada vez más, nuevos acercamientos para estudiar y comprender la comunicación desde las formas en que los actores sociales participan en su sociedad. Los escenarios de la migración, particularmente la migración de jóvenes, viene replanteando retos teóricos-metodológicos para poder interpretar las nuevas formas de participar en su sociedad como ciudadanos. Este artículo parte de un estudio cualitativo (bajo la perspectiva metodológica de complementación con etnografía y entrevistas a profundidad) sobre la migración juvenil en Tijuana, con el objeto de redimensionar las distintas formas en que este sujeto se coloca frente a dinámicas estructurales para participar e incorporarse como ciudadano. Lo que se presenta es una parte de los resultados que, a partir de un mapa de las representaciones subjetivas de los actores jóvenes sobre la ciudad fronteriza $y$, desde la comunicación como procesos intersubjetivos, se ve que la construcción de imaginarios de futuro en la migración es una huella que está marcando en buena medida en los procesos fronterizos.

Palabras clave: Comunicación, ciudadanía cultural, migración juvenil, Tijuana.

\footnotetext{
* Maestro en comunicación por el ITESo. Profesor de tiempo completo UABC Tijuana. Catedrático desde 1998 en la misma institución en las áreas de metodología de la investigación y teoría sociocultural de la comunicación. Ha participado en investigaciones nacionales y locales sobre comunicación y cultura, estudios de recepción de medios y consumo cultural. Ha publicado artículos de investigación en el libro La revolución también es una calle (UIA Tijuana) en la revista Texto Abierto (UIA León), xiII Anuario coneIcc, Revista electrónica Razón y palabra, la Revista colombiana Periferia, Revista electrónica entre otros. Correo electrónico: yayo_leon@yahoo.com
} 
Contemporary social life requires more and more the use of new approaches to study and understanding communication from the point of view of the actors participating in their society. The scenarios of migration, particularly youth migration, pose theoretical and methodological challenges to the interpretation of new forms of participation in society. This article is based on a qualitative study (from the methodological perspective of complementation with ethnography and in-depth interviews) of youth migration to Tijuana with the goal of re-dimensionalizing the different forms in which the subject faces various structural dynamics to participate and incorporate himself as a citizen. We present part of the results that, parting from a map of the subjective representations of the young actors about the border city, and from the point of view of communication as an intersubjective process, we see how the construction of imaginaries of the future in migration is a factor that is affecting in an important measure the border processes.

Key words: Communication, cultural citizenship, youth migration, Tijuana.

\section{COMUNICACIÓN Y SOCIEDAD CONTEMPORÁNEA:}

UN ACERCAMIENTO SOCIOCULTURAL PARA RE-PENSAR AL ACTOR SOCIAL

El tránsito de un estado de sociedad medieval a un estado de sociedad moderna ha generado no únicamente una sociedad distinta, sino también nuevos actores sociales y nuevas formas de ser parte de este mundo. Pero no sólo eso, hay espacio heurístico que nos acerca a la comprensión de la vida social desde un ángulo distinto, la comunicación, una novedosa manera para una taquigrafía de lo social (Ortiz, 2004) ya que tenemos herramientas de interpretación de la realidad muy distintas a las de otros tiempos. Antes del siglo XX, en pocos o casi nulos espacios sociales se hablaba de comunicación. Hoy es un término común en distintos órdenes de la vida social, desde el espacio cotidiano donde una familia se preocupa por sus "formas" de comunicación, hasta las maneras en que distintos grupos culturales tratan de comprenderse en complejos escenarios de interacción, intersubjetivos y mediáticos (Thompson, 1998), esto es, pensar en comunicación, y específicamente en el estudio de la comunicación, nos instala en un debate que hoy en día, obligadamente, tiene dos frentes: el estudio de la comunicación desde la esfera mediática; y la compresión de la comunicación desde los procesos intersubjetivos de interacción social. Podemos decir que ambos están implicados, pues si la comunicación es pensada desde procesos donde los medios colectivos de comunicación son centrales, ésta no 
puede dejar de lado a la comunicación interpersonal o intersubjetiva donde se tejen múltiples sentidos y significados, y, a su vez, la comunicación intesubjetiva no puede estudiarse independientemente del contexto sociocultural y mediático en el orden global.

En los espacios académicos ha predominado el estudio del fenómeno comunicativo massmediático, por lo que proponemos un primer recorte epistémico con el abordaje de lo comunicacional más allá de los medios, y entendemos a los procesos intersubjetivo como los cuales son eje de producción y reproducción la vida social. La comunicación es la plataforma de toda interacción social que toma sentido al ponerse en común entre dos o más actores sociales. El estudio de la comunicación desde este ángulo "desplaza epistemológica y metodológicamente el foco de análisis" sobre los procesos de construir y compartir socialmente significados, reubicando el foco de interpretación en los "sujetos sociales y los procesos de producción de sentido" (Fuentes, 1999). Por lo tanto, la perspectiva sociocultural, como propone Fuentes Navarro, nos parece un recurso analítico que entiende a la comunicación como un proceso que se teje de manera compleja como en el Esquema 1:

EsQuema I

La comunicación como proceso sociocultural

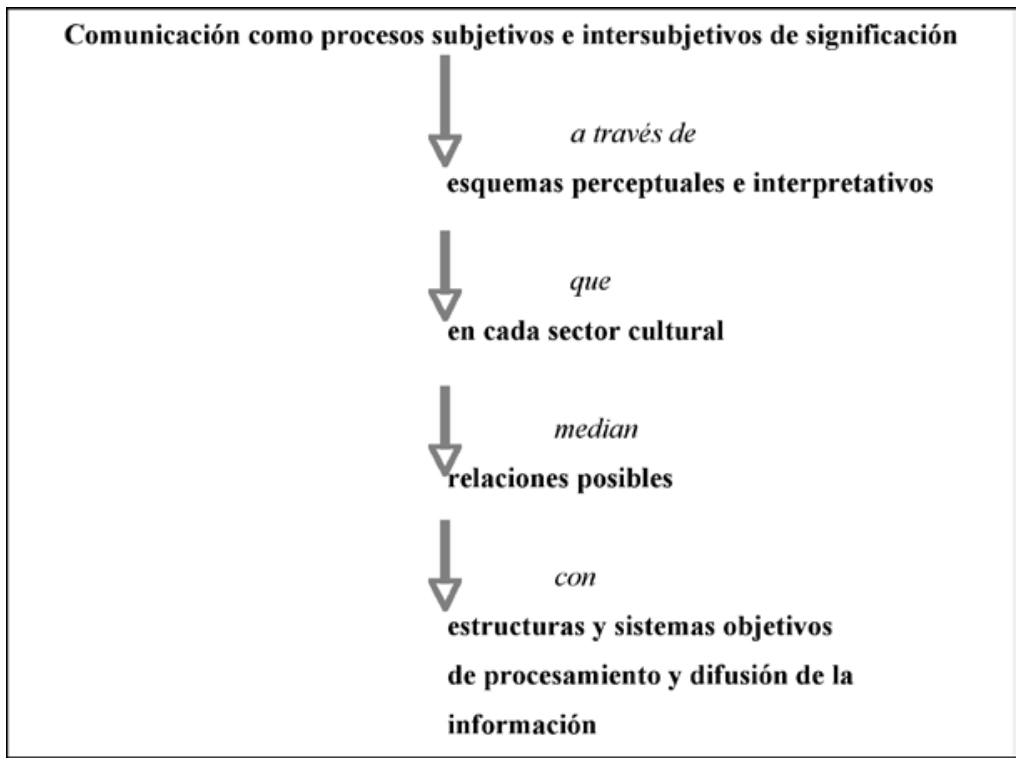

Fuente: Fuentes Navarro, R., 1999

Comunicación y migración juvenil: $\bullet 217$ un imaginario de futuro en Tijuana 
El esquema nos permite ver que el asunto de la comunicación está soportado en la esencia misma de las prácticas de los actores, esto es, que este proceso de interacción y prácticas intersubjetivas ocupa -y debe ocupar- un lugar importante en los enfoques y acercamientos científicos sociales sobre el sujeto y su capacidad de agencia.

De este modo, la diversidad de actores sociales de nuestro tiempo como mujeres, hombres, jóvenes, adultos, consumidores, ciudadanos, empleados, migrantes, manifestantes, indígenas, niños, por solo mencionar algunos, tienen la posibilidad de ser pensados y estudiados desde la comunicación. Esto interpela teórica y metodológicamente la manera en cómo se estudia la comunicación, por lo que el reto se encuentra en saber "penetrar hermenéuticamente" (Reguillo, 1996; 1998) en las formas a través de las cuales los sujetos sociales -específicos y situados social e históricamente-, llevan a cabo infinitas formas de generar procesos intersubjetivos en lo interpersonal, grupal y colectivo.

Alejandro Grimson (2001) plantea que la comunicación está compuesta de experiencias y prácticas que los actores sociales llevan a cabo como "organización de la experiencia y de la acción humana por medios simbólicos”. Esto apela a una interpretación de la sociedad y la cultura desde sus modos de hacer, de convivir, de percibir, de sońar en relación con las condiciones que nos rodean y con lo cual establecemos una relación en la vida como personas, grupos, colectividades. Grimson propone que "si comunicar es poner en común", se admite que ese acto es una interacción de dos o más personas en una situación ubicada histórica y socialmente, misma que sólo es posible a través de sentidos y significaciones compartidos y/o diferenciados.

La comunicación intersubjetiva involucra entonces la existencia y la producción de un código compartido y de una diferencia. La construcción de las fibras del entramado simbólico -o estructuras de significación - de ciertas prácticas sociales son resultado, pero a su vez recurso de formación de sentidos posibles que se dan en procesos de larga duración, dentro de un tiempo-espacio concreto, compartido e imaginado, y que es el componente, la base, el suelo de esos sentidos comunes y de las prácticas cotidianas.

\section{Comunicación y migración juvenil}

Proponemos pensar la cuestión objetiva de la migración juvenil a la frontera como una acción sociocultural en su dimensión comunicacional, en tanto en ella se llevan a cabo prácticas de comunicación con un espesor cultural importante por su carácter subjetivo, hecho de percepciones, representaciones e imaginarios 
que los actores hacen sobre el proceso migratorio. Esto plantea que estamos quizá más cerca de tener elementos de interpretación más sistematizados para comprender algo que a principios del siglo XX nos asombraba: que somos distintos tipos de sujetos, capaces de conocer y reconocer, de interpretar y reinterpretar, pero sobre todo, capaces de actuar en un mundo que por mucho no es tan predecible, o al menos eso parece en este contexto de globalización.

\section{UN CONCEPTO COMO SISTEMA DE EXPLORACIÓN ABIERTO:}

El actor social lo entendemos como lo suficientemente capaz de expresar y poner en acción una serie de destrezas y habilidades durante su vida en este mundo, que van más allá de la noción de "definición y protección" como elementos medulares del concepto clásico de ciudadanía. Para ello colocamos de manera central a la ciudadanía cultural como nuestra categoría analítica para el análisis de la migración, lo que nos permite reubicar las dimensiones clásicas de ciudadanía como la social, política y civil, en tanto éstas se conformaron desde una relación circunscrita a las entidades Estado-sujeto fundamentalmente.

La cultura es la matriz que nos permite ver cómo los universos simbólicos de los actores sociales que migran activan los recursos que sus condiciones socioculturales específicas permiten; por lo tanto, la ciudadanía es una práctica sociocultural que no se arraiga a un espacio definido o a un solo tipo de institución, si no que se practica desde la gestión de recursos y acciones de los sujetos al poner en práctica sus propias soluciones a través de diferentes estrategias posibles por un habitus específico (Bourdieu, 1990).

Renato Rosaldo ha incorporado esta dimensión analítica al estudio del referente espacial, pero no únicamente a lo nacional, sino que también incluye a lo local en prácticas de "afiliación, derecho e influencia estrechamente ligados a minorías o grupos socioculturales específicos” (Rosaldo, 2000). La noción cultural de ciudadanía, a manera de concepto articulador, interpreta en su dimensión empírica cómo ciertos grupos, conservando o negociando particularidades y diferencias culturales, traban relaciones de poder para incorporarse a una sociedad.

La ciudadanía cultural permite el entendimiento de las formas de resolución de formas de vida en desiguales condiciones sociales, culturales, políticas y económicas; se lleva a cabo mediante una serie de estrategias o prácticas específicas para ser parte de normas y dinámicas de una sociedad dominante. Decimos

Comunicación y migración juvenil: • 219 un imaginario de futuro en Tijuana 
entonces que en la búsqueda del reconocimiento a la pertenencia se pone en acción, y es ahí, en la acción misma, donde el actor social liga profundamente su "experiencia personal y lo que se percibe en el todo social" (Aceves, 1997) con el objeto de dar sentido a esa forma de llevar a cabo los deseos de pertenencia, no sólo legal. En este marco, la ciudadanía cultural nos permite conocer bajo qué imaginarios se construye la pertenencia y cómo se relaciona con el uso de territorios y espacios, como práctica que los sujetos llevan a cabo desde sus "matrices culturales" (Martín-Barbero, 1987) y como dispositivos de resistencia social y diferenciación social. El lugar se convierte en el espacio público en el que se ejerce la apropiación simbólica del territorio, misma que confecciona el entramado sociocultural definiendo y dibujando las condiciones de vida social.

Rossana Reguillo (2000a) afirma que la ciudadanía es una dinámica de lo social que se concreta, se desarrolla y se posibilita en la práctica, esto es, hablamos de una concepción no sólo de ciudadanos que asumen obligaciones y tienen acceso a derechos con el simple acto de ser parte de una nación, sino más bien que la acción tiene un peso cultural importante.

La migración como práctica social es un lugar metodológico clave para el análisis cultural que intenta dar luces para conocer cómo y desde dónde se conforman los tipos de reapropiación territorial en procesos diaspóricos, y que a manera de "prácticas de espacio" son un elemento constitutivo de las culturas de la migración, pues es la "invención del territorio" y su concepción de la vida donde es posible generar la reorganización geopolítica del mundo, que también se expresa en la apropiación -con sus estrategias - de nuevos espacios. (De Certeau, 1997; Reguillo, 2000b).

Esto nos remite esencialmente a re-pensar y dimensionar la capacidad de agencia como la capacidad de movilizar recursos materiales y simbólicos a fin de trasformar la realidad, sobre todo cuando se emprende la búsqueda de pertenencia y reconocimiento desde los derechos de género, de clase, de edad, sexuales, raciales y migratorios, como una forma genuina de intentar ser parte de una sociedad que ofrece pocas posibilidades de desarrollo en contextos de incertidumbre.

\footnotetext{
${ }^{1}$ Entendemos por "matrices culturales" los espacios, tiempos, escenarios y actores que conforman una estructura de significación que el sujeto social lleva consigo a manera de impronta social, y que remite al "lugar" de pertenencia sociocultural en el cual se ha desarrollado, ha interactuado y ha formado una visión y valoración del mundo. Estos "anclajes” tienen dos dimensiones, las profundas y las situacionales; las profundas se refieren a las marcas socio-históricas; las situacionales a los ámbitos y escenarios en los que el sujeto participa e interacciona.
} 
La cuestión de la ciudadanía cultural se construye en una arena de conflicto, contención, negociación y reacomodos a modo de proceso dialéctico, donde el eje organizador de esa relación son condiciones de hegemonía y subalternidad desde el sentido sociológico gramsciano (González, 1990). El análisis desde la ciudadanía cultural se entiende como un proceso de producción de nuevas formas culturales que da la posibilidad de pertenecer a los que han estado en diferentes niveles de exclusión, pero es también un recurso analítico "policéntrico" (Reguillo, 2003a) que nos aproxima al significado que nutre a diferentes grupos sobre la resolución de los derechos de reconocimiento y diversas formas incorporación; al tiempo que nos permite ver las maneras en que los sujetos activan sus anclajes profundos en función de imaginarios de futuro en la relación con la estructura social (véase, Esquema 2).

Para efectos de esta propuesta, entendemos la pertinencia de la ciudadanía cultural por que nos acerca a mirar a la migración como una forma de poner en acción percepciones del mundo vivido, rescatando el tránsito existente entre el desarraigo territorial y cultural, y el proceso de adaptación sociocultural que experimenta un migrante, por ejemplo. Este aspecto, que ocurre en el terreno de lo simbólico, se debe entender como una forma genuina de escenificar el derecho cultural a buscar cumplir con un imaginario de futuro y también a reconocerse frente a los otros con quienes se encuentra.

La migración, en cualquiera de sus patrones (intrarregional o extrarregional) es una cuestión que está marcando nuestra contemporaneidad, y es un proceso complejo y multidimensional, pero que es importante describir en términos del sentido que se le construye a las maneras de hace valer el derecho a buscar otras formas de vida.

EsQuema 2

Lugar de definición y acción de la ciudadanía cultural

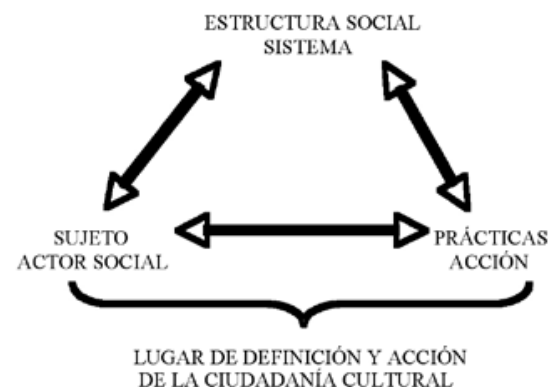

Fuente: elaboración propia

Comunicación y migración juvenil: $\bullet 22 \mathrm{I}$ un imaginario de futuro en Tijuana 


\section{ETHNOSCAPE JUVENIL.}

\section{LOS SUJETOS EMPÍRICOS Y EL ABORDAJE METODOLÓGICO}

El antropólogo Arjun Appadurai (1990) discute los elementos centrales del fenómeno contemporáneo proponiendo un marco analítico desde el cual problematiza cinco cuestiones que están reflejando la complejidad del engranaje global. Utilizando la metáfora de "paisaje" (o landscape) en tanto es una noción que le permite entender el carácter irregular, diferenciado, desigual y dinámico que cada individuo experimenta según el tipo de flujo en el que se ubica: "paisaje de personas", el "paisaje de medios", el "paisaje de tecnología", el "paisaje de dinero" y el "paisaje de ideas". Los cinco paisajes nos parecen un referente fundamental en el análisis de los flujos humanos, pero es el punto de la desterritorialización al que refiere el ethnoscape el que nos lleva a poner el acento en la condensación y en las proporciones, no menores, de personas que viven fuera de su lugar de origen. Los ethnoscapes (o paisaje de personas), para Appadurai, representan la categoría que describe el movimiento -o flujo- de personas como un fenómeno que describe algunos aspectos de la globalización.

Los inmigrantes, turistas, refugiados, exiliados, trabajadores temporales, representan la experiencia de un movimiento que hoy en día no es exclusivo de grupos relativamente privilegiados, sino de sujetos que por varias razones tienen que resolver situaciones de condiciones de vida.

\section{Lo juvenil y la migración}

La migración de jóvenes en el patrón migratorio intrarregional creció fuertemente entre las décadas de 1970 y 1980, teniendo como telón de fondo las profundas alteraciones sociopolíticas de países latinoamericanos en esas décadas. Para 1980, había 335 mil jóvenes que vivían en países distintos a los de origen, y para 1990, había 350 mil. No hay un repunte fuerte, pero sí es significativo, lo que nos lleva pensar que la migración intrarregional si bien es cuantitativamente considerable y pausada, es mucho menor a la que presenta la migración extrarregional (Martínez Pizarro, 2000).

El patrón migratorio latinoamericano extrarregional tiene como característica esencial el tener como destino Estados Unidos. Este patrón masivo se ha intensificado cuantitativamente, representando uno de los más dinámicos en las últimas décadas. Entre 1980 y 1990 la población joven migrante en los Estados Unidos se elevó hasta casi 1.6 millones (originarios, principalmente de México y Centroamérica), alrededor del $22 \%$ del total de migrantes en este 
país. Como telón de fondo se presentan las enormes diferencias en los niveles de desarrollo de Estados Unidos frente a los países latinoamericanos. En este sentido, se nos hace pertinente indicar que, para los jóvenes que se inscriben en este flujo migratorio, el tener como opción migrar a este país, pesa mucho en la toma de decisiones para el proyecto futuro por la posibilidad de acceder a mejores oportunidades laborales, de formación personal y la "consecución de logros como metas culturales" (Martínez Pizarro, 2000). Lo local, lo nacional y lo global toman hoy otras dimensiones, y los jóvenes emergen como actores sociales protagonistas y que dinamizan estos cambios.

Una posible hipótesis a este incremento la podríamos comprender en el cruce de dos vectores. Por un lado, el vector del imaginario de las expectativas de vida, si consideramos que los jóvenes, en cuanto categoría de análisis amplia e imprecisa, se ubican en una fase de vida en la que se encuentran o estarán dentro del campo laboral, lo que los hace sujetos en circunstancias socioculturales concretas, que deben de tomar decisiones para conseguir objetivos y metas que se construyen a través de su imaginario de futuro. Y esto nos remite al otro vector que, en el caso particular de México, se cruza con la percepción valoral sobre su entorno, esto es, para los jóvenes mexicanos lo que se encuentra con un imaginario sobre lo que les gusta de su país por las oportunidades de vida que ofrece, representa una desventaja frente a otras valoraciones. Por ejemplo, sólo un $10.4 \%$ en mujeres y un $10.2 \%$ de hombres mexicanos les gusta su país por las oportunidades de vida que se presentan, lo cual contrasta con un $58.1 \%$ de mujeres y $57.6 \%$ de hombres que les gusta su país por su cultura y tradiciones (Reguillo, 2001).

La gran diferencia entre uno y otro indicador nos deja apreciar las profundas marcas que dejan las actuales condiciones de vida del país, y que aparecen como poco óptimas para la construcción de un proyecto futuro, lo que nos permite fortalecer la hipótesis sobre un quiebre entre las expectativas de vida y su relación con las motivaciones por lo cuales la migración aparece como una opción de vida.

\section{El marco de lo juvenil, otra mirada}

Entrar en la discusión de la temática de juventud es un reto que implica ubicar un marco de comprensión preciso, y a su vez, crear una plataforma analítica desde la cual se toma posición para pensar a este sector social. Es importante comprender la situación de los jóvenes en distintos momentos y contextos, por lo que se hace necesario trazar un mapa desde el cual pensamos y entendemos la condición de joven. 
Un primer acercamiento lo hacemos tomando distancia de la definición más estereotipada de este sector social, y es de desde esta ruptura desde la que decimos que definir al sujeto joven a partir de su condición biológica no es suficiente. Esto nos hace saber que, más allá de pensar que un sujeto, el ser joven, representa una forma temporal y de cambio biológico por el cual se le puede identificar. El asunto de la edad física marca un espacio de comprensión para esta etapa de vida, pero no nos permite profundizar en aspectos en los cuales se configuran al actor joven a partir del lugar que distintas sociedades le asignan socio-históricamente. La categoría de joven, por tanto, desde la perspectiva cronológica, es limitada y nos ubica sólo en la dimensión biologicista dejando fuera la dimensión biográfica o de trayectoria de vida social.

Nos adscribimos a la noción de lo juvenil o de juventud representada como un segmento de vida social y como constructo que "refieren a relaciones sociales históricamente situadas y representadas" (Valenzuela, 1997). Esto nos marca dos cuestiones, que el concepto de juventud nos puede llevar a nociones contradictorias y limitadas si no se plantea con precisión y rigor, y dos si no se toman en cuenta la heterogeneidad social y la diversidad de modalidades con las que hace presencia el sector joven en una sociedad determinada. Así, afirmamos que cada tiempo-espacio socio-histórico elabora su propia noción del "ser joven".

Lo anterior nos lleva a retomar tres dimensiones que José Antonio Pérez Islas (2004) propone para pensar en una noción moderna de juventud:

a) La segmentariedad lineal: que refiere a los "episodios de las trayectorias" de vida. Aquí es importante hacer visibles cómo las formas y los sistemas de producción-reproducción social de una sociedad prefiguran un tipo de sujeto joven.

b) La segmentariedad circular: círculos, ámbitos o entornos personales, regionales y globales, y que regularmente se extienden con respecto al sujeto joven desarrollando identidades e imaginarios sociales.

c) La segmentariedad binaria: dimensión que alude a la relaciones de oposición de la esfera estructural y simbólica dependencia.

La juventud es una construcción social y un concepto relacional que depende del reconocimiento de las relaciones establecidas en lo social, y del significado que le asignan los marcos culturales y las representaciones colectivas que operan como mediaciones. Lo que nos hace ver que la noción debe abordarse tomando en cuenta las matrices culturales como formatos que demarcan la relación con la 
estructura, es decir, la interrelación entre instituciones sociales, sujetos -en nuestro caso jóvenes- y las prácticas en la vida social. Estos elementos nos colocan en una perspectiva centrada en las formas de los procesos sociales más amplios, y que nos permite esgrimir la limitante conceptual biologicista y esencialista.

Frente a todo lo anterior, podemos decir que para estos tiempos, la conceptualización del sujeto joven no puede circunscribirse a lo estrictamente biológico o quedar en una noción que homogeniza la experiencia juvenil, pues desde la perspectiva sociocultural estas definiciones son más acotaciones que herramientas heurísticas. Ser joven tiene implicaciones socioculturales diferentes en distintos contextos porque sus esquemas de representación y sus prácticas culturales están ubicados en espacios sociales y simbólicos diferenciados y desiguales. Rossana Reguillo (2003), en un estado de la cuestión sobre jóvenes y estudios culturales afirma que no se ha logrado tener un mapa lo suficientemente profundo sobre dos asuntos clave:

a) Pensar lo juvenil como problemática que toque "la multiplicidad diacrónica y sincrónica" de las experiencias de este grupo social.

b) Las tendencias de estudio se han desarrollado centralmente en las "formas de agregación, adscripción y organización juvenil” que se generan desde dos frentes "al margen" o "en contradicción" con las entidades institucionales con las que se relacionan.

Lo anterior nos remite indiscutiblemente a cuestionarnos sobre las construcciones analíticas de los actores jóvenes regularmente visibles, esto es, pensados desde las identidades o culturas juveniles. Pero la pregunta por el actor joven "no institucional" sigue teniendo un marco de análisis que se ha desarrollado de modo limitado. Los estudios de los jóvenes "no incorporados" al sistema social hegemónico son más reducidos en cuanto a la pregunta central por la relación que guarda este sujeto con la estructura social. Es relativamente escaso lo que se sabe sobre este tipo de acercamientos, sobre todo de las formas de participación y construcción de futuro en procesos de migración.

\section{El abordaje metodológico}

La configuración metodológica es una relación interactiva entre artilugios tecnológicos y decisiones de investigación que van dando forma a un proceso que tiene diferentes fases, estrategias, niveles y plataformas de la exploración para aprehender algún aspecto de la realidad.

Comunicación y migración juvenil: $\bullet 225$ un imaginario de futuro en Tijuana 
Nos parece importante recuperar la idea de que la migración contiene una dimensión simbólica que requiere ser estudiada y revalorada en los estudios de comunicación por la generación de sentidos y significados en sus formas de representación social que los sujetos hacen cuando dejan su ciudad para incorporarse a otra. Para ello, nuestro marco epistémico o interrogativo partió de la premisa de buscar responder sobre el significado sociocultural que los actores jóvenes migrantes le asignan a la puesta en escena de sus estrategias de construcción de "imaginarios de futuro" en el espacio fronterizo tijuanense.

El estudio tiene como sujetos-objeto de estudio a los jóvenes migrantes, los cuales aparecen como protagonistas en procesos de migración en estos tiempos. El espacio de tiempo tiene dos niveles, desde donde se trazó el mapa de acercamiento para el trabajo de campo. El primero es que los actores jóvenes migrantes hayan tenido como mínimo 5 años de residencia en la ciudad, en tanto el referente de la llegada a la ciudad tiene un proceso de reconocimiento y hay un proceso de adaptabilidad a lo que es Tijuana como ciudad. El segundo nivel tiene que ver con el momento mismo del trabajo de campo, que se realizó en el segundo trimestre del 2006, lo que nos ubicó en ciertos escenarios que eran referentes sociales de fondo que los informantes tenían en su percepción de muchas cuestiones relacionadas con el haber llegado a esta ciudad.

El proceso de migración que describimos desde la experiencia juvenil es fundamentalmente la migración interna o interregional, lo que nos colocó en el planteamiento metodológico de trabajar con informantes mexicanos que hayan tenido como lugar de partida alguno de los estados de la República Mexicana, por lo que tenemos que el espacio central de nuestras interrogantes es Tijuana como el lugar privilegiado de acogida.

Metodológicamente nos orientamos por las categorías teórico-analíticas desarrolladas en el capítulo I y II, mismas que fueron el principio de nuestro programa metodológico que se desarrolló bajo tres ejes que se articularon de manera transversal en la construcción de los datos:

1) Construcción de imaginarios de futuro en la migración

2) Apropiación y prácticas de espacio en la ciudad de Tijuana

3) Construcción y conformación de ciudadanía

Para ello decidimos implementar una estrategia metodológica combinada con dos métodos y técnicas de investigación pertinentes para nuestro objeto de estudio, y generar información de carácter empírico de esta forma: la entrevista 
a profundidad, que tiene carácter de herramienta central, nos ayudó a conocer las significaciones sobre lo que implica para un joven migrar a una ciudad como Tijuana, entendiendo que desde lo discursivo el sujeto joven toma posición desde las matrices culturales que lo ubican en su experiencia y en su relación con el resto de la sociedad en la que interacciona; la observación participante fue la herramienta complementaria pues fue la estrategia de acompañamiento, reflexividad y registro de todo lo que aconteció dentro del proceso de acercamiento con los informantes así como de la realización de las entrevistas. Esto nos dio cuenta de algunos rasgos de las prácticas que están alrededor de los migrantes en su condición juvenil en el territorio fronterizo.

Se realizaron 12 entrevistas en dos dimensiones. En la primera dimensión se abordó una estructura temática de cuatro ejes (véase, Cuadro 1), que fue diseñada bajo los criterios analíticos que articulan nuestra pregunta de investigación:

Cuadro I

Ejes temáticos de entrevistas

\begin{tabular}{|l|l|}
\hline a) Anclajes situacionales y profundos \\
\hline b) Apropiación y experiencia en el territorio fronterizo \\
\hline c) Construcción de imaginarios de futuro en la migración \\
\hline d) Construcción y práctica de su ciudadanía \\
\hline
\end{tabular}

La segunda dimensión permitió profundizar en los elementos temáticos que, durante la primera parte, emergieron y se consideraron como importantes para regresar a ellos. De esta manera, pudimos realizar las entrevistas combinando dos tipos, la entrevista semi-estructurada y la entrevista a profundidad, con la siguiente muestra de informantes.

IMAGINARIO DE FUTURO Y TIJUANA COMO ESPACIO LIMINAL

La comunicación es una dimensión de lo social, y como tal, es una práctica organizadora, generadora, estructuradora de otras prácticas, en las que el sujeto social interacciona con otros sujetos en un tiempo-espacio socialmente construido y constituido.

Asumimos, para el caso de haber analizado la migración juvenil a Tijuana, que las estructuras y las acciones sociales son elementos socioculturales que están en continua transformación, pues se reconfiguran, sobre todo si no perdemos de vista la escala macrosocial donde la globalización está generando

Comunicación y migración juvenil: $\bullet 227$ un imaginario de futuro en Tijuana 
un sin números de pautas y transformaciones a nivel micro, como estilos de vida y las maneras en cómo se posiciona el actor joven en su mundo social.

\section{Aspiraciones en el espacio: metas e imaginarios de futuro}

Tijuana representa, desde el discurso de los jóvenes entrevistados, la ciudad donde se redefinen elementos centrales de su configuración como sujetos sociales, donde el eje central de esta redefinición son las formas en que el proceso migratorio transforma las coordenadas socioculturales. Esto es, la toma de decisión de venir a esta ciudad no es fortuita, y responde a una serie de cuestiones socioculturales donde los diferentes grupos juveniles que llegan a la ciudad de Tijuana la construyen como un escenario que es a la vez punto de llegada y punto de partida, en el sentido de que se llega y se parte para emprender las estrategias para cumplir el horizonte de futuro, influyendo en la reproducción social dentro de marcos de incertidumbre para el logro de aspiraciones e imaginarios de futuro. Tijuana como frontera es un espacio social fundamental en la forma en que estos jóvenes construyen, modelan y perfilan sus formas de vida y la conformación de una identidad. Aunque en todas las entrevistas el tema de lo fronterizo no se refiere a la situación de llegar para cruzar, sino llegar para establecer aquí las rutas de vida que constituirán ese horizonte de futuro. La apropiación del espacio de la ciudad de Tijuana, rompe con las interpretaciones en donde la vida fronteriza de Tijuana se reduce a lugar de paso o cruzar el bordo.

Podemos decir que para nuestro caso, los informantes se adscriben inicialmente a pensar a Tijuana como el punto de llegada, es el referente del espacio simbólico y el espacio objetivo para la constitución de sus objetivos de vida, veamos:

Pues Tijuana me parece una ciudad, a los años que yo llegué era diferente, pero pues para mí Tijuana es un lugar, pues una buena ciudad, una buena ciudad por todo lo que es su trabajo, por todo lo que te ofrece como persona. Tú tienes la alternativa, tomas la buena, para ser mejor persona, o tomas la mala, por el mal camino... Tijuana es una ciudad que te deja ser. $^{2}$

A Víctor le parece de esta manera:

A Tijuana muchos vienen nada más a hacer dinero y se regresan. Es un círculo que me da risa, porque vienen y están 5 a 6 meses, hacen dinero y se van y se regresan

${ }^{2}$ Entrevista con Reyna.

228 • Gerardo León Barrios 
los 6 meses. Se gastan tanto dinero, vuelven a juntar un poquito de dinero, en lo que está en el campo allá para el pasaje y se regresan los 6 meses. Ese es un aspecto que no me llama mucho. No tengo visa ni pasaporte, y mis hermanos tienen, y pues para mí el mundo, mi realidad es Tijuana y no tanto el otro lado. Como ciudad fronteriza se me hace interesante, lo que tiene que ver con el flujo de otras ciudades del país y otros estados, el que Tijuana tenga esa, esa riqueza de otras culturas, luego aprendes más de otras partes...

Para Antonio significa de esta manera:

Tomé la decisión de llegar aquí a Tijuana porque yo oía que aquí en Tijuana sí había manera de hacerla, de salir adelante, más que en otras partes, porque está pegado en la frontera, pero nunca, nunca me pasó por la mente pasarme para el otro lado, salir de México y me voy de Tijuana. Nos decían, desde niños, que Tijuana estaba muy bonito, muchas cosas a diferencia de otros lugares... aunque ahora se escucha bastante que la droga, que desmadre, muertos. Tijuana es una ciudad bonita, tranquila, como la viva uno. ${ }^{4}$

Para los jóvenes migrantes, la ciudad, a manera de mapa mental, representa una profunda transformación a sus esquemas de percepción y a la construcción de su identidad, pues como definimos anteriormente, uno de los elementos centrales de la constitución de la migración es el espacio-territorio, en este caso los actores jóvenes han tenido que resignificar este elemento por una ciudad distinta a la de origen. El joven migrante se divide entre lo que para él tiene sentido como espacio del lugar de origen, y el nuevo espacio.

Cuando llego a Tijuana veo una ciudad bastante rural, comparado con la ciudad donde yo nací. Hoy, en muchas zonas de la ciudad, hay pobreza, se nota poca educación. Pero se ven posibilidades. Posibilidades muchas, a pesar de todo, el mayor atractivo para mí es que era que es una ciudad donde puedes realizar tus sueños o tratar de perseguirlos, porque tienes mayores posibilidades de hacerlos en una ciudad donde no está tan explotado, que en otro lugar donde lo que hagas, vas a tener 20 ó 30 competencias, gente que va hacer lo mismo que tú, como en el D.F. Por ejemplo, en el empleo, cuando yo llego a esta ciudad mi intención era obtener experiencia en mi profesión, posteriormente se me dio la chamba. Pero

\footnotetext{
${ }^{3}$ Entrevista con Víctor

${ }^{4}$ Entrevista con Antonio
}

Comunicación y migración juvenil: 229 un imaginario de futuro en Tijuana 
veo que tengo beneficios, como joven preparado, sobre otros lugares o estados, por las condiciones económicas de nuestra ciudad. Ahora estoy tratando de desarrollar algunos proyectos, emprender proyectos. Creo que la idea de muchos jóvenes en esta ciudad que es alcanzar una estabilidad económica, tus sueños, no veo que se puedan ya hacer en otros lados. ${ }^{5}$

Juan lo ve desde esta perspectiva:

Pues en Tijuana me siento bien, bien a gusto, mi meta es... yo pienso que unos tres años, poder echar el techo de mi casa. En Tijuana busco mi meta, que es llegar a tener para echarle madera y colar, pero pues lo que necesita uno es trabajo, y así yo pienso que sí, pos del tiempo que yo tengo para acá, puedo decir, ya pude comprarme un carro, tener mis herramientas, tener mi casa. Yo pienso que voy bien, en cuatro años que he estado acá. Yo pienso que sí puedo... Por eso Tijuana, en algún sentido, se puede hacer logros. Aunque allá en Veracruz está muy bonito, cuando voy pues ver a mi mamá, mis hermanos, les da gusto. Entonces, veo que mi trabajo en Tijuana, para mí, digamos, es todo, así como me decían que era este lugar, que había mucho trabajo, que se ganaba más o menos, y pues esa era la idea cuando me iba a venir, y pues aprovechamos, me parece bien patrabajar, para vivir pos, no muy bien todo está caro, hasta la tierra..., pero me gusta que puedes conseguir dónde emplearte. ${ }^{6}$

El punto aparentemente es simple, pero implica una serie de transformaciones en la estructura de las acciones, de la agencia. La espacialización se construye en los espacios y territorio inmediatos, y al haber una cambio del signo de identificación, el mapa mental tiene que reconstruirse generando estrategias, prácticas y maneras de ver lo que les rodea en el espacio social "nuevo" o diferente. Esto nos lleva a entender cómo se han "estructurado" formas de interacción social en la ciudad a partir de la construcción y reconstrucción de significados que contrastan y que los actores sociales generan para tomar decisiones en la vida cotidiana, como lo esquematizamos de la siguiente manera:

De las ciudades de origen:

1. Para el caso de D.F., se recuperan las experiencias de la gran ciudad, las posibilidades de tener acceso a un sin número de ofertas: trabajo, educativas,

\footnotetext{
${ }^{5}$ Entrevista con Ricardo
}

${ }^{6}$ Entrevista con Juan

230 • Gerardo León Barrios 
esparcimiento; sin embargo, frente a ello Tijuana sigue teniendo acceso a otras opciones como el consumo.

2. Para el caso de otras ciudades, se asumen con lugares de profundo arraigo e identidad por sus características de ciudades pequeñas, las cuales quedan libres de delincuencia, violencia y drogas.

3. Marcadamente, la ciudad de origen se sigue reconociendo como el "lugar" de origen, con todo lo que ello implica: la familia, los amigos, las experiencias tempranas, los aprendizajes.

4. Pese a un fuerte contraste en lo estético con la "fealdad" de Tijuana, las ciudades de origen no compensan el problema del desempleo, en donde no se puede tener la accesibilidad a un mercado de trabajo para poder pensar en el "desarrollo" como joven.

5. Las ciudades de origen son un referente para comparar.

De Tijuana:

1. Tijuana se reconoce como la ciudad por excelencia de "oportunidades" laborales.

2. Tijuana es una ciudad que, siendo fea, se compensa por la facilidad de poder acceder a una "calidad de vida" que de otra forma, en las ciudades de origen no se podría conseguir o se tardaría años en lograrlo, a esto se agrega el buscar vivir en la ciudad cuando los índices delictivos se perciben como altos.

3. Tijuana, si bien tiene cercanía con Estados Unidos, no se ve como la ciudad de paso para "pasar" al otro lado. Se reconoce que la economía del vecino país, tiene efecto en la economía de Tijuana.

4. Tijuana tiene una diversidad cultural muy marcada. Esto se ve como un elemento que a la vez trae conflictos, pero también bondades.

Si bien las prácticas sociales son una forma de experimentar de modos diversos la ciudad, porque es ahí donde se construyen los significados en relación a su identidad. Por ello, el sujeto construye relaciones con personas de la sociedad con la finalidad de integrarse a la dinámica de la ciudad.

Tijuana, desde que llegué y desde antes, es un lugar en el país de los más importantes económicamente, es un país con una cultura distinta y muy rica de la que te puedes encontrar en cualquier otra parte del país. Por lo mismo, por la diversidad, por las personas que chocamos aquí, todos traemos un poquito de nuestro lugar de origen

Comunicación y migración juvenil: • $23 \mathrm{I}$ un imaginario de futuro en Tijuana 
y Tijuana es una ciudad en crecimiento pero, pero a cantidades industriales... ahora me he topado con mucha gente de Chiapas, de otros lugares que antes no se sabía. Como que se pasa la voz, como que se pasa el rumor por el país de que Tijuana es un lugar donde puedes crecer... Así que pues yo veo a la ciudad como parte de mí, por ejemplo la 5 y 10 , se me hace que hay de todo un poco... cuando yo voy para allá, que me gusta mucho andar ahí caminando, atravesando el puente, y ves al Licenciado que es muy reconocido, luego ves artículos originales y artículos piratas, entonces, hay de todo en la 5 y 10 , hay asaltantes, hay gente invidente, hay de todos los lugares de la república, y para mí eso es Tijuana, una ciudad muy transitada, muy ágil y, creo, que soy una persona también muy ágil, en ese aspecto.?

En la medida que el sujeto joven realiza actividades en la ciudad, las apropia haciéndolas parte de su nuevo contexto social. El joven migrante crea estrategias cotidianas de integración al momento de establecer relaciones de convivencia con otros grupos a través de la interacción, los actores sociales "negocian" y se "apropian" del espacio urbano, y a su vez lo transforman convirtiéndolo en un espacio de significación (Hannerz, 1986), como también podemos ver desde la historia personal de Elena:

(...) por ejemplo, yo recuerdo mucho los lugares donde estuve y eran verdes ¿̨no?, entonces voy a parques o a lugares que tiene muchos árboles, donde hay un café, un lugar donde yo puedo estar platicando y pasándomela bien divertida, teniendo cosas donde pueda ejercitarme, jsimón!, ese es mi forma de verme en este lugar, aunque en Tijuana no hay ese lugar ideal, lo tratas de hacer tuyo... voy con mis amigos o con mi novio a $i$ El porkys!, en Plaza Fiesta. Me gusta porque siento que es relajado, no se necesita andar bien arreglada. Me gusta mucho el Cecut, porque como paso por ahí, mi trabajo está cerca, entonces, de repente me echo un vistazo para ver las exposiciones... por ahí es donde paso más cuando tengo tiempo libre, me alimento de lo tijuanense... ${ }^{8}$

El joven migrante apropia los significados de las actividades del espacio. Los usos sociales que el joven migrante hace de la ciudad van a definir el fenómeno social mediante un sistema de comunicación que remite a las "maneras de hacer". Se establece en la ciudad en condiciones de precarias y de desventaja

\footnotetext{
${ }^{7}$ Entrevista con Alberto.

${ }^{8}$ Entrevista con Elena.
}

232 • Gerardo León Barrios 
social. Por ello, las relaciones que establezca con otras gentes de la ciudad le otorgan apoyo.

Un aproximación interpretativa sería que se teje una relación con la ciudad y se constituye rasgos identitarios a partir de esa interacción, que son las formas de apropiación e incorporación al espacio tijuanense. El significado que esto tiene afirma que los trazos de las prácticas de usar la ciudad, los discursos desde los que se vislumbra y se divide el espacio urbano en esta frontera, son fundamentales para las formas de re-configurar la identidad como joven en este terreno (véase, Esquema 3).

\section{Esquema 3}

Imaginario de futuro en el espacio social de Tijuana

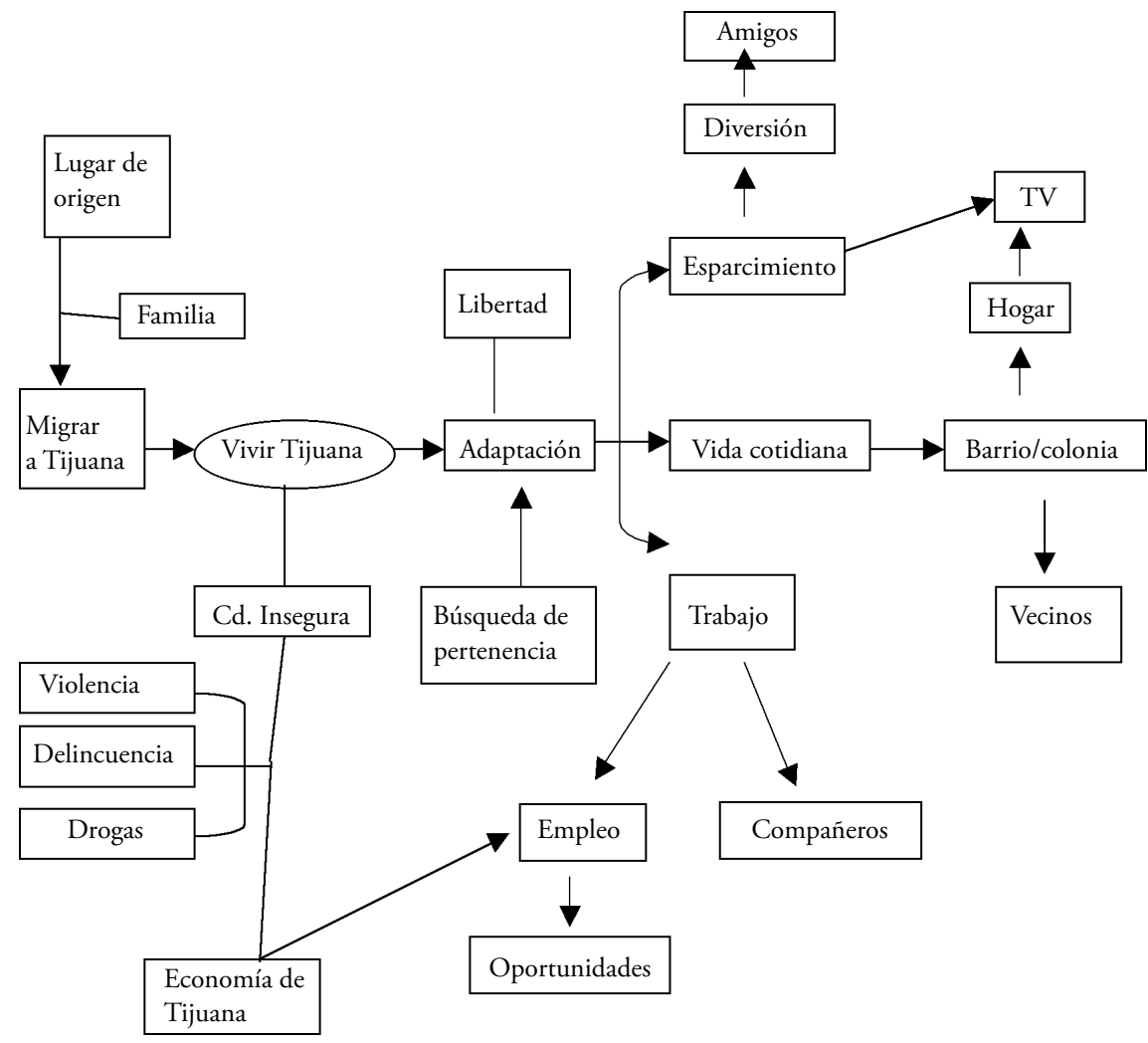

Comunicación y migración juvenil: $\bullet 233$ un imaginario de futuro en Tijuana 
El imaginario social que corresponde a la noción de espacio-territorio resulta importante puesto que éste nos permite ver las formas de cristalizar elementos de los imaginarios sociales anteriores (la condición juvenil y la migración juvenil).

La migración a Tijuana parte de dos cuestiones. Primeramente, que el proceso de decisión implica un reordenamiento tanto con el lugar de origen como con las relaciones de familia. Por otro lado, el migrar a Tijuana asume que la ciudad es conflictiva por sus problemas de inseguridad.

Sin embargo el elemento central es la "adaptabilidad" como formas de apropiación de la ciudad.

Pues yo me relaciono con la ciudad por lo que hago, por donde ando con amigos, con personas que conozco y que son del mismo lugar, por el Fundadores, ahí vive mucha gente que son del mismo pueblo que yo. Entonces, voy allá y siento como que estoy más cerca de Sinaloa, entonces me siento identificado, hay mucha gente y el mismo modo de hablar. Me siento como que estoy, más tranquilo en la ciudad, mi adaptación es más tranquila, más en confianza con la gente. Y en otras partes no, como que si pasa un extraño no le hablas...?

Con Hortensia el ángulo de adaptación a Tijuana, es de esta manera:

Cuando estoy sola, tengo que hacer tareas, veo televisión o escucho música, leo mmm... limpio mi casa, y voy con mis amigos, nos gusta ir mucho al cine. Vamos a eventos del Cecut, de vez en cuando, pero la mayor parte de mi tiempo estoy sola, pero me cuido. Allá en Tepic tienen una idea de Tijuana como la ciudad de la muerte, narcos, prostitutas, horrible la ciudad. Pero cuando llegué aquí mi círculo en el que empecé a moverme fue muy distinto. La mayoría de las personas que vienen a Tijuana no vienen a estudiar. Y uno tiene que ver como se van acomodando tu vida, tus actividades, tus rutinas en una ciudad como ésta, es una ciudad muy grande con muchísima inversión, gente, cosas que se hacen..., de repente te puede asustar eso, tanta gente, tanto ruido. Esa, esa es la sensación que me da, es una ciudad grande, una ciudad industrializada, pero hay muchísimo más... cómo decirlo, más posibilidades, porque es más grande. ${ }^{10}$

\footnotetext{
${ }^{9}$ Entrevista con Jorge, Op. cit

${ }^{10}$ Entrevista con Hortensia, $O p$. cit.

234 • Gerardo León Barrios
} 
Desde este ángulo, existe un sentimiento de caos en cuanto infraestructura, tráfico, las afluencias de gente de un lado a otro, clima de inseguridad, es para los tijuanenses migrantes un lugar que se le puede encontrar el gusto por vivir, entre el desorden y orden. Entendida, entonces, como una ciudad significativa por su ductibilidad para adaptarse por el trabajo, ofertas educativas, convivir con otras personas, dan la confianza de que se puede vivir aprovechando las oportunidades de todo tipo. La vecindad con Estados Unidos, se asume como el elemento que le da ese toque de lugar estratégico para construir las rutas de los horizontes de futuro y sostenerlos.

\section{NotAS FinALES}

Lo que tenemos en este panorama es que los jóvenes que han migrado a la ciudad de Tijuana han crecido y desarrollando sus expectativas de vida bajo un contexto de reordenamientos sociales, políticos, económicos y culturales (de tradiciones, instituciones, códigos morales y prácticas legítimas y formas específicas de llevar a cabo acciones), lo que los ha ubicado en la necesidad de reconfigurar sus formas de identificación con Instituciones Sociales y con el Estado, esto es, desde su individualidad y su capacidad de agencia han transformado sus formas y estilos de vida, con cambios importantes en la estructura social. Siguiendo a Giddens, Turner y otros (1991), entendemos que la migración no únicamente es la acción de salir del lugar de origen, comprende acciones y condiciones históricas y sociales específicas en las que los actores jóvenes:

a. Deciden migrar

b. Emigran

c. Se reubican en el nuevo espacio-territorio

Esto es un proceso social que está conformado, además de actos individuales, por la compleja relación que hay entre el contexto social, político, económico y cultural y las acciones que este sector de la sociedad, en su condición de migrantes, tejen desde su agencia y con la estructura social, y es, en esta relación, desde donde se reproduce la vida sociocultural. De esta manera, vemos que la acción social de la migración, percibida en un nivel sociocultural micro, se relaciona con la estructura o los sistemas sociales ubicados en un nivel de análisis macro. El análisis, en el sentido giddeniano, no se coloca en los polos micro o macro, si no que recupera prácticas sociales a fin de tener el foco de interpretación en la relación entre la acción y la estructura, donde ambos

Comunicación y migración juvenil: $\bullet 235$ un imaginario de futuro en Tijuana 
elementos los pensamos implicadamente, esto es, la acción de migrar refiere a una estructura social específica, y la estructura social específica tiene la huella de la acción social de migrar.

La ciudadanía cultural parece que puede ser una respuesta para el análisis para explorar formas de generar prácticas socioculturales como las que los jóvenes llevan a cabo para poder incorporarse a un sistema social y conseguir sus derechos básicos como trabajo, educación, ingresos, salud, esparcimiento, calidad de vida, lo implica que se tienen que poner en acción otros recursos como ciudadanos, aunque quizá no resulten en movilizaciones o agregaciones que busquen contrarrestar el estado de las cosas en las que se vive, para el caso de este estudio.

El cambio de territorio transforma imaginarios sociales tradicionales que se construyen en el lugar de origen, como lo es el horizonte de futuro, que quizá puede estar o no consciente, pero la migración a Tijuana trae otros elementos para las conformaciones identitarias.

Las huellas de la incertidumbre son parte de ese horizonte de futuro, y cada joven, como lo hemos explorado en este estudio, busca aproximarse a sus diversas formas de vivir la experiencia de la migración. El asunto es clave para poder entender la compleja relación que se teje entre el sistema social, el sujeto joven y la acción o prácticas, donde la dimensión intersubjetiva de la comunicación se coloca como aspecto fundamental y como reto académico para profundizar en diversas formas ejecutar y modelar formas de vida, si bien, estos sujetos jóvenes pasarán a las cifras de los sujetos adultos que estarán en otro tipo de relación con la estructura social.

\section{REFERENCIAS}

Aceves Lozano, J. (1997). "Ciudadanía ampliada. La emergencia de la ciudadanía cultural y ecológica”, en Razón y palabra, Número 5, Año 1, diciembre-enero 1996-97, en http://www.razonypalabra.org.mx/

Appadurai, A. (1996). La modernidad desbordada. Dimensiones culturales de la globalización. FCE, Buenos Aires, 2001.

Bourdieu, P. (1990). Sociología y cultura. México: Grijalbo-CNCA.

De Certeau, M. (1997). La invención de lo cotidiano. 1 Artes de hacer. México: Universidad Iberoamericana, ITEso y Centro Francés de Estudios Mexicanos y Centroamericanos.

Fuentes Navarro, R. (1999). "Perspectivas socioculturales postdisciplinarias en la investigación de la comunicación”. En G. Orozco. Lo viejo y lo nuevo. Investigar la comunicación en el siglo XXI. España: Ediciones de la Torre. 
Giddens, A. et al. (1991). La teoría social, hoy. México: conaculta-Alianza.

González Sánchez, J. (1990). Sociología de las culturas subalternas. México: Universidad Autónoma de Baja California.

Grimson, A. (2001). Interculturalidad y comunicación. Buenos Aires: Norma.

Martín Barbero, J. (1987). De los medios a las mediaciones. Comunicación, cultura, hegemonia. México: Gustavo Gili.

Martínez Pizarro, Jorge (2000). Migración internacional de jóvenes latinoamericanos y caribeños: protagonismo y vulnerabilidad. Santiago de Chile: CEPAL/CELADE.

Ortiz, R. (1997). Mundialización y cultura. Buenos Aires: Alianza Editorial.

Pérez Islas, J. A. (2004). "Historizar a los jóvenes. Propuestas para buscar los inicios". En I. Pérez y M. Urteaga. Historias de los jóvenes en México. Su presencia en el siglo XX. México: Instituto Mexicano de la Juventud.

Reguillo, R. (1996). La construcción simbólica de la ciudad. Sociedad, desastre y comunicación. Guadalajara: ITESO.

Reguillo, R. (1998). "De la pasión metodológica o de la (paradójica) posibilidad de la investigación”. En R. Mejía y Sandoval, S. Tras las vetas de la investigación cualitativa. México: ITESo.

Reguillo, R. (2000). Cuatro ensayos de comunicación y cultura para pensar lo contemporáneo, Conferencia inaugural, Maestría en Comunicación con Especialidad en Difusión de la Ciencia y la Cultura. uia-León/ıтeso. México.

Reguillo, R. (2000). Emergencia de culturas juveniles. Estrategias del desencanto, Argentina: Editorial Norma.

Reguillo, R. (2001). "La gestión del futuro. Contextos y políticas de representación”, en JÓVENES, año 5, No. 15. México: Instituto Mexicano de la Juventud.

Reguillo, R. (2001). "Jóvenes y esfera pública. Cartografía de la cultura política de los jóvenes mexicanos”, 1a. Encuesta Nacional de Juventud. México: Instituto Mexicano de la Juventud.

Reguillo, R. (2003). "Jóvenes y estudios culturales. Notas para un balance reflexivo". En J. M. Valenzuela. Los estudios culturales en México. México: FEC.

Reguillo, R. (2003). "Políticas de representación y desafíos culturales. La visibilidad de América Latina”, en Renglones, No. 53. México: ITEso.

Reguillo, R. (2003). "Ciudadanía cultural. Una categoría para pensar en los jóvenes", en Renglones, No. 55. México: ITEso.

Rosaldo, R. (1999). "Ciudadanía cultural, desigualdad, multiculturalidad”, en El bordo: retos de frontera. No. 3. México: UIA Tijuana.

Rosaldo, R. (2000). "La pertenencia no es un lujo: procesos de ciudadanía cultural dentro de una sociedad multicultural". En Desacatos \#3. México: ciesas.

Thompson, J. (1993). Ideología y cultura moderna. Teoría crítica social en la era de la comunicación de masas. México: UAM-Iztapalapa.

Valenzuela Arce, J. M. (1997). "Culturas juveniles, identidades transitorias. Un mosaico para armar”. En JÓVENES, nueva época, año 1, núm. 3. México: Causa Joven.

Comunicación y migración juvenil: • 237 un imaginario de futuro en Tijuana 